\title{
Re: predictors of surgical sperm retrieval in non-obstructive azoospermia: summary of current literature
}

\author{
Giovanni M. Colpi ${ }^{1,2}$ (1) $\cdot$ Ettore Caroppo ${ }^{3}$ (1)
}

Received: 1 June 2020 / Accepted: 3 June 2020 / Published online: 11 June 2020

(c) Springer Nature B.V. 2020

It is well known that patients with azoospermia due to spermatogenic dysfunction, the so-called non-obstructive azoospermia (NOA), may still have residual foci of intact spermatogenesis in their testes, and that if testicular sperm are retrieved, they can be utilized in ICSI to enable patients to father their genetically own children. Since retrieving testicular sperm represents the most relevant step before treatment, many efforts have been spent in the past years to identify pre-surgical parameters able to predict the presence of sperm in the testes.

In the current issue of "International Urology and Nephrology", Arshad et al. have performed an accurate and thorough review of the literature in this field [1]. They have found that Klinefelter syndrome (KS) and Sertoli cell-only (SCO) histopathology were associated with reduced sperm retrieval success, while a testis volume higher than $12.5 \mathrm{ml}$, history of cryptorchidism, and the use of microdissection testicular sperm extraction (microTESE) as surgical technique to retrieve testicular sperm and hormonal treatments were associated with better sperm retrieval rates. Nevertheless, none of the predictors, alone or combined, could predict with great accuracy the chances of successful sperm retrieval (SSR) in patients with NOA.

It is undoubtful that microTESE increases the chance of SSR in patients with NOA when compared to the other surgical techniques. Confirming the findings of the unique randomized-controlled trial published to date on this matter [2], a meta-analysis investigating the performance of the available surgery to harvest testicular sperm in patients with

Giovanni M. Colpi

gmcolpi@yahoo.com

1 Andrology Unit, Procrea Institute, 6900 Lugano, Switzerland

2 Andrology and IVF Unit, Clinica San Carlo, 20037 Paderno Dugnano, Milano, Italy

3 Asl Bari, Department of Maternal and Child Health, Reproductive and IVF Unit, PTA "F. Jaia", 70014 Conversano, Bari, Italy
NOA showed that microTESE was 1.5 times more likely to result in SSR as compared with the conventional TESE (cTESE), which, on the other hand, it was two times more likely to result in SSR as compared with testicular aspiration (TESA) [3]. Since patients with NOA may show intratesticular heterogeneity of spermatogenesis, with few intact (i.e., with normal caliber) seminiferous tubules surrounded by tubules of variable thinness, depending on the degree of the spermatogenic damage, only microTESE can allow the microscopical identification and selection of the still normal tubules, looking large and opaque as compared to the surroundings, while reducing the complications and minimizing tissue loss. We have previously demonstrated that the identification of the so-called "dilated" seminiferous tubules at high magnification during microTESE predicts SSR with a good diagnostic accuracy (AUC 0.89), correctly classifying $82.4 \%$ of cases and explaining $60 \%$ of the variability of SSR; dilated tubules contained sperm in $90 \%$ of cases, while the random excision of non-dilated tubules was successful in terms of sperm retrieval only in $7 \%$ of cases. An excellent diagnostic accuracy (AUC 0.93) in predicting SSR was obtained when testis histology was added to the model [4].

This raises another interesting point, that is the impact of testis histology on the chance of SSR. The present study shows that SCO histopathology was associated with reduced sperm retrieval success, which confirms the previous findings in the field. A previously available testis histology report may be of great value in managing patients with NOA: indeed, microTESE has been found to be critical for increasing the chance of SSR in patients with SCO [5, 6]. However, the authors correctly report that a prior diagnostic testicular biopsy may have detrimental effects on the likelihood of SSR in further surgery aiming at sperm retrieval, so that a fragment of testicular tissue should be obtained for histology only during therapeutical surgery. The removal of a small fragment of testicular tissue representative of the appearance of most of the parenchyma during microTESE allows the correct scoring of spermatogenesis according to 
the classically defined five main histological patterns, which have been demonstrated to perform better than the Johnsen's score in predicting sperm retrieval, since different stages of spermatogenesis may coexist as mixed pattern in the same sample [7]. In addition, testis histology may enable the identification of intratubular germ cell neoplasia in situ (GCNIS) as previously recommended [7].

While intraoperative features can predict SSR with a great accuracy, there are a few pre-operative conditions that can reliably predict the presence of sperm in the testis. Among these, there is great consensus that history of cryptorchidism is associated with better chances of SSR in men with NOA. A study utilizing an artificial neural network (ANN) to model the chance of SSR of 1026 men with NOA (770 training set, 256 test set) undergoing microTESE found that cryptorchidism was significant to the model [OR 2.29 (1.47-3.57), $p<0.0001$ ] [8]. It is not clear why patients with NOA and history of cryptorchidism may have better chances of sperm retrieval compared to those with idiopathic NOA. A retrospective study performed on 225 men with NOA and history of cryptorchidism showed that SSR rate was similar in men with history of unilateral or bilateral cryptorchidism, and was not affected by the age at surgery; however, patients with undetectable inhibin B levels were less likely to have SSR [9].

The authors report that SSR may be lower in patients with KS compared to non-KS counterparts. This is still a debated issue, with studies reporting opposite results. A prediction model performed on 1026 men with NOA showed that history of KS was associated with increased chances of SSR in microTESE [7]. It has to be pointed out, as the authors correctly observe, that SSR in KS may be greatly affected by some factors like age and serum testosterone level, so that heterogeneity of study populations makes synthesizing studies' results difficult.

Neither hormonal parameters nor testis volume may help in individuating patients with NOA with better chances of sperm retrieval. It has been previously demonstrated that serum FSH level does not predict SSR in patients with NOA: on the contrary, a large sample study (792 subjects) provided the counterintuitive demonstration that men with high FSH levels had better chance of sperm retrieval compared to men with FSH below $15 \mathrm{mIU} / \mathrm{ml}$ [10]. Similarly, viable sperm have been found even in men with nearly atrophic testes, if microTESE is used [11]. Heterogeneity in surgical procedures of sperm retrieval may justify differing results, such as in a recent meta-analysis [12], where a significant association of higher testis volume $(>12.5 \mathrm{ml})$ with better sperm retrieval outcomes was found. In addition, the different methods of testis volume estimation (Prader orchidometer vs ultrasound measurements, and the variability of the latter according to the volumetric formulas used) could have introduced additional bias to the analysis.
The authors also evaluated the possible role of adjuvant medical and surgical treatments in improving sperm retrievals in men with NOA. While there is quite a consensus that men with hypogonadism should have their testosterone optimized before attempting surgery, the literature is unable to provide definitive results about the role of hormonal treatment in eugonadal men. An interesting study demonstrated, however, that treatment with follicle-stimulating hormone (FSH) was able to improve the expression of androgen receptor on Sertoli cell (SCAR), and that SCAR elevation after treatment was strongly associated with SSR [13]. Further studies are warranted to replicate and extend these findings.

Recently, studies have been sought to evaluate the mRNA expression of testis-specific genes in the seminal plasma as candidate predictors of residual spermatogenesis in men with NOA [14, 15]. Although the results look promising, further studies in this field are warranted.

In conclusion, the study of Arshad et al. confirms that SSR in men with NOA cannot be fully predicted by pre-surgical markers. Indeed, many factors, including the surgeon skill and experience (particularly when microTESE is used) [16], different tissue processing methods, and the time, the skill, and effort, dedicated to the identification of spermatozoa in the testicular specimen may greatly affect the sperm retrieval rates [17]. Based on these results, no man with $\mathrm{NOA}$, apart from the few with deletion of the AZFa, AZFb, or $\mathrm{AZFa} / \mathrm{b}$ regions of the $\mathrm{Y}$ chromosome, should be discouraged from attempting surgery aiming at sperm retrieval: however, the best surgical treatment should be offered to patients to maximize their chances of SSR.

\section{References}

1. Arshad MA, Majzoub A, Esteves SC (2020) Predictors of surgical sperm retrieval in non-obstructive azoospermia: summary of current literature. Int Urol Nephrol. https://doi.org/10.1007/s1125 5-020-02529-4

2. Colpi GM, Colpi EM, Piediferro G, Giacchetta D, Gazzano G, Castiglioni FM, Magli MC, Gianaroli L (2009) Microsurgical TESE versus conventional TESE for ICSI in non-obstructive azoospermia: a randomized controlled study. Reprod Biomed Online 18:315-319

3. Bernie AM, Mata D, Ramasamy R, Schlegel PN (2015) Comparison of microdissection testicular sperm extraction, conventional testicular sperm extraction, and testicular sperm aspiration for nonobstructive azoospermia: a systematic review and meta-analysis. Fertil Steril 104:1099-1103

4. Caroppo E, Colpi EM, Gazzano G, Vaccalluzzo L, Piatti E, D'Amato G, Colpi GM (2019) The seminiferous tubule caliber pattern as evaluated at high magnification during microdissection testicular sperm extraction predicts sperm retrieval in patients with non-obstructive azoospermia. Andrology 7:8-14

5. Deruyver Y, Vanderschueren D, Van der Aa F (2014) Outcome of microdissection TESE compared with conventional TESE in nonobstructive azoospermia: a systematic review. Andrology 2:20-24 
6. Esteves SC, Ramasamy R, Colpi GM, Carvalho JF, Schlegel PN (2020) Sperm retrieval rates by micro-TESE versus conventional TESE in men with non-obstructive azoospermia-the assumption of independence in effect sizes might lead to misleading conclusions. Hum Reprod Update. https://doi.org/10.1093/humupd/ dmaa006 (online ahead of print)

7. Dohle GR, Elzanaty S, van Casteren NJ (2012) Testicular biopsy: clinical practice and interpretation. Asian J Androl 14(1):88-93

8. Ramasamy R, Padilla WO, Osterberg C, Srivastava A, Reifsnyder JE, Niederberger C, Schlegel PN (2013) A comparison of models for predicting sperm retrieval before microdissection testicular sperm extraction in men with nonobstructive azoospermia. J Urol 189:638-642

9. Barbotin AL, Dauvergne A, Dumont A, Ramdane N, Mitchell V, Rigot JM, Boitrelle F, Robin G (2019) Bilateral versus unilateral cryptorchidism in nonobstructive azoospermia: testicular sperm extraction outcomes. Asian J Androl 21:445-451

10. Ramasamy R, Lin K, Gosden LV, Rosenwaks Z, Palermo GD, Schlegel PN (2009) High serum FSH levels in men with nonobstructive azoospermia does not affect success of microdissection testicular sperm extraction. Fertil Steril 92:590-593

11. Bryson C, Ramasamy R, Sheehan M, Palermo GD, Rosenwaks Z, Schlegel PN (2013) Severe testicular atrophy does not affect the success of microdissection testicular sperm extraction. J Urol 191:175-178

12. Corona G, Minhas S, Giwercman A, Bettocchi C, Dinkelman-Smit M, Dohle G, Fusco F, Kadioglou A, Kliesch S, Kopa Z, Krausz C, Pelliccione F, Pizzocaro A, Rassweiler J, Verze P, Vignozzi L, Weidner W, Maggi M, Sofikitis N (2019) Sperm recovery and ICSI outcomes in men with non-obstructive azoospermia: a systematic review and meta-analysis. Hum Reprod Update 25:733-757

13. Kato Y, Shiraishi K, Matsuyama H (2014) Expression of testicular androgen receptor in non-obstructive azoospermia and its change after hormonal therapy. Andrology 2:734-740

14. Hashemi MS, Mozdarani H, Ghaedi K, Nasr-Esfahani MH (2020) Could analysis of testis-specific genes, as biomarkers in seminal plasma, predict presence of focal spermatogenesis in non-obstructive azoospermia? Andrologia 52(2):e13483

15. Pansa A, Sirchia SM, Melis S, Giacchetta D, Castiglioni M, Colapietro P, Fiori S, Falcone R, Paganini L, Bonaparte E, Colpi G, Miozzo M, Tabano S (2014) ESX1 mRNA expression in seminal fluid is an indicator of residual spermatogenesis in nonobstructive azoospermic men. Hum Reprod 29(12):2620-2627

16. Ishikawa T, Nose R, Yamaguchi K, Chiba K, Fujisawa M (2010) Learning curves of microdissection testicular sperm extraction for nonobstructive azoospermia. Fertil Steril 94:1008-1011

17. Ramasamy R, Reifsnyder JE, Bryson C, Zaninovic N, Liotta D, Cook C-A, Hariprashad J, Weiss D, Neri Q, Palermo G, Schlegel PN (2011) Role of tissue digestion and extensive sperm search after microdissection testicular sperm extraction. Fertil Steril 96(2):299-302

Publisher's Note Springer Nature remains neutral with regard to jurisdictional claims in published maps and institutional affiliations. 